\title{
A data acquisition system for real-time magnetic equilibrium reconstruction on ASDEX Upgrade and its application to NTM stabilization experiments
}

\author{
L.Giannone, ${ }^{\mathrm{a}}$, M.Reich ${ }^{\mathrm{a}}$, M.Maraschek ${ }^{\mathrm{a}}$, E.Poli ${ }^{\mathrm{a}}$, C.Rapson ${ }^{\mathrm{a}}$, L.Barrera ${ }^{\mathrm{a}}$, \\ R.McDermott ${ }^{\mathrm{a}}$, A.Mlynek ${ }^{\mathrm{a}}$, Q.Ruan ${ }^{\mathrm{b}}$, W.Treutterer ${ }^{\mathrm{a}}$, L.Wenzel ${ }^{\mathrm{b}}$, A.Bock ${ }^{\mathrm{a}}$, \\ G.Conway $^{\mathrm{a}}$, R.Fischer ${ }^{\mathrm{a}}$, J.C.Fuchs ${ }^{\mathrm{a}}$, K.Lackner ${ }^{\mathrm{a}}$, P.J.McCarthy ${ }^{\mathrm{c}}$, R.Preuss ${ }^{\mathrm{a}}$, \\ M.Rampp ${ }^{\text {d }}$, K.H.Schuhbeck ${ }^{\mathrm{a}}$, J.Stober ${ }^{\mathrm{a}}$, H.Zohm ${ }^{\mathrm{a}}$, and ASDEX Upgrade \\ Team $^{\mathrm{a}}$ \\ ${ }^{a}$ Max Planck Institute for Plasma Physics, EURATOM Association, 85748 Garching, \\ Germany \\ ${ }^{b}$ National Instruments, Austin, TX 78759-3504, Texas, USA \\ ${ }^{c}$ Department of Physics, University College Cork, Association EURATOM-DCU, Cork, \\ Ireland \\ ${ }^{d}$ Computing Centre (RZG) of the Max Planck Society and the Max Planck Institute for \\ Plasma Physics, 85748 Garching, Germany
}

\begin{abstract}
The pre-emptive stabilization of a neoclassical tearing mode, NTM, requires the calculation of the tokamak magnetic equilibrium in real-time. A launcher mirror is positioned to deposit electron cyclotron current drive on the rational surface where the NTM should appear. A real-time Grad-Shafranov solver using constraints from magnetic probe, flux loop and Motional Stark Effect measurements has been developed to locate these rational surfaces and deliver this information to the mirror controller in real-time. A novel algorithm significantly reduces the number of operations required in the first and second step of the solver. Contour integrals are carried out to calculate the $q$ profile as a function of normalized radius and the rational surfaces are found by spline interpolation. A cycle time of $0.6 \mathrm{~ms}$ for calculating two tokamak equilibria in parallel using four current basis functions with magnetic constraints only and using six current basis functions with magnetic and MSE constraints has been achieved. Using these tools, pre-emptive stabilization
\end{abstract}

Email address: Louis.Giannone@ipp.mpg.de (L.Giannone) 
of a $m / n=3 / 2$ NTM mode in ASDEX Upgrade could be demonstrated.

\section{Introduction}

Neoclassical tearing modes (NTMs) are a major limitation to high beta tokamak operation in H-mode scenarios. The suppression of a NTM with electron cyclotron current drive (ECCD) to improve tokamak performance by raising the operational limits on poloidal beta, $\beta_{p}$, has been demonstrated in many tokamak experiments $[1,2,3,4,5,6]$. Previously a ramp in the toroidal magnetic field, a rigid plasma displacement or a scan of the launching antenna were used to place ECCD deposition at the rational $q$ surface of the NTM. A search and suppress algorithm, seeking to minimize the amplitude of the NTM measured by external magnetic probes, was developed to demonstrate feedback controlled stabilization of the NTM [7]. The tracking of the normalized radius of the NTM can be realized by locating the phase jump in temperature fluctuations at the NTM frequency measured by the electron cyclotron emission diagnostic (ECE) $[6,8,9]$. Stabilization of a NTM by the feedback controlled movement of a mirror on JT-60U [6] and ASDEX Upgrade [10] has been demonstrated.

In this paper, pre-emptive stabilization of a NTM in ASDEX Upgrade using the magnetic equilibrium from a real-time Grad-Shafranov solver to feedback control a launching mirror will be presented. Real-time magnetic equilibria are required for the microwave beam tracing code, TORBEAM [11], to calculate the mirror angle necessary for depositing ECCD on the rational surface where the NTM is located. Pre-emptive NTM stabilization has been demonstrated on JT-60U [12] and DIII-D [13]. The safety factor profile, $q(r)$, is calculated from the flux surface contour integrals at ten values of normalized poloidal flux. Rational surfaces can then be located as a function of normalized radius by spline interpolation. The line integrated phase shift of five chords of a DCN laser interferometer are processed using the flux surfaces to provide an electron density profile by Abel inversion in real-time 
[14]. The normalized radii of the rational $q$ surfaces, the electron density profile and the poloidal flux matrix are sent over the real-time reflective memory network to the node running the TORBEAM simulations. The mirror angle for ECCD deposition, including microwave refraction caused by caused by density and magnetic field inhomogeneity, is then calculated.

The feedback controlled movement of a mirror for stabilizing a single NTM without the need for real-time magnetic equilibrium reconstruction and microwave beam tracing using six channels of inline ECE has been demonstrated in a proof of principle experiment $[15,16]$. However, such a system requires temperature fluctuations for locating the NTM and therefore pre-emptive NTM stabilization is not possible. The proposed system with real-time magnetic equilibrium reconstruction and microwave beam tracing would also be able to stabilize multiple NTM modes without the installation of an inline ECE system in the waveguide of each gyrotron.

In Section 2, the details of the procedure for calibrating the magnetic probes and flux loops in ASDEX Upgrade is summarized. The real-time Grad-Shafranov solver for calculating the magnetic equilibrium constrained to fit 40 magnetic probes and 18 flux loop differences, is presented in Section 3. The solver is based on an innovative algorithm using discrete sine transforms and a tridiagonal solver that realizes an equilibrium poloidal flux matrix on a $33 \times 65$ grid. The real-time calculation of the $q$ profile by contour integration and the poloidal beta, $\beta_{p}$, and plasma inductance, $l_{i}$ from Shafranov integrals is discussed in Section 4. The extension of the real-time Grad-Shafranov solver to include constraints on the current profile in the plasma core from the Motional Stark Effect, MSE, diagnostic is discussed in Section 5. In Section 6, the data acquisition system is presented and the cycle time benchmarks for the real-time magnetic equilibrium calculations are summarized. The application of the real-time Grad-Shafranov solver to pre-emptive NTM stabilization experiments in ASDEX Upgrade is presented in Section 7. 


\section{Vacuum field calibration}

The reconstruction of magnetic equilibria in ASDEX Upgrade relies on an accurate test of the parameters assumed for the position and orientation of the magnetic probes and flux loops and their calibration factors. The simplest test, carried out in the first shot of the day, is to ensure that the integrators are delivering the expected response within an allowed error range to the flow of currents in individual poloidal field coils. The calculation of the magnetic field at a radius, $r$, and height, $z$, in response to a current loop of radius a begins with the expression for the Green's function for the poloidal flux per steradian, $\psi[17,18,19]$ :

$$
\psi=\frac{\mu_{o} I}{2 \pi} \sqrt{\left.(a+r)^{2}+z^{2}\right)}\left[\left(1-k^{2} / 2\right) K\left(k^{2}\right)-E\left(k^{2}\right)\right]
$$

where $\left.k^{2}=4 a r /\left((a+r)^{2}+z^{2}\right)\right), K\left(k^{2}\right)$ is the complete elliptic integral of the first kind and $E\left(k^{2}\right)$ is the complete elliptic integral of the second kind $[20,21]$. The derivatives in the $Z$ and $R$ direction are the radial and vertical magnetic field components [19] :

$$
\begin{aligned}
& b_{r}=-\frac{1}{r} \frac{\partial \psi}{\partial Z}=\frac{\mu_{o} I}{2 \pi r} \frac{z}{\beta \alpha^{2}}\left(\left(a^{2}+r^{2}+z^{2}\right) E\left(k^{2}\right)-\alpha^{2} K\left(k^{2}\right)\right) \\
& b_{z}=\frac{1}{r} \frac{\partial \psi}{\partial R}=\frac{\mu_{o} I}{2 \pi r} \frac{r}{\beta \alpha^{2}}\left(\left(a^{2}-r^{2}-z^{2}\right) E\left(k^{2}\right)+\alpha^{2} K\left(k^{2}\right)\right)
\end{aligned}
$$

where $\alpha^{2}=(\mathrm{a}-\mathrm{r})^{2}+\mathrm{z}^{2}$ and $\beta^{2}=(\mathrm{a}+\mathrm{r})^{2}+\mathrm{z}^{2}$.

A detailed optimization process is carried out once a year to ensure that the assumed geometry and orientation of the magnetic probes, the position of the poloidal field coils, the calibration factors of the poloidal field currents and the calibration factors of magnetic probe and flux loop integrators are self consistent. A current pulse in each poloidal field coil is produced with a flat top of sufficient duration so that currents excited in the passive stabilization loop no longer flow. All the other poloidal field coils are open circuit 
so that no current flow in them is possible. The separate excitation of the toroidal field coil allows magnetic probe measurements due to misalignment to be compensated. Such a procedure was executed to perform an accurate calibration of the magnetic probes on the TCV tokamak [22]. The minimization of the least squares difference of the predicted and measured signals was reduced to a steepest descent problem. A self consistent set of calibration factors for calculating magnetic equilibria on ASDEX Upgrade is obtained [23]. In Fig. 1 is an overview of the poloidal field coils of ASDEX Upgrade. The position and orientation of the magnetic probes on ASDEX Upgrade are shown in Figs. 2 and 3 and the location of the pairs of flux loops that measure the flux loop differences are shown in Fig. 4.

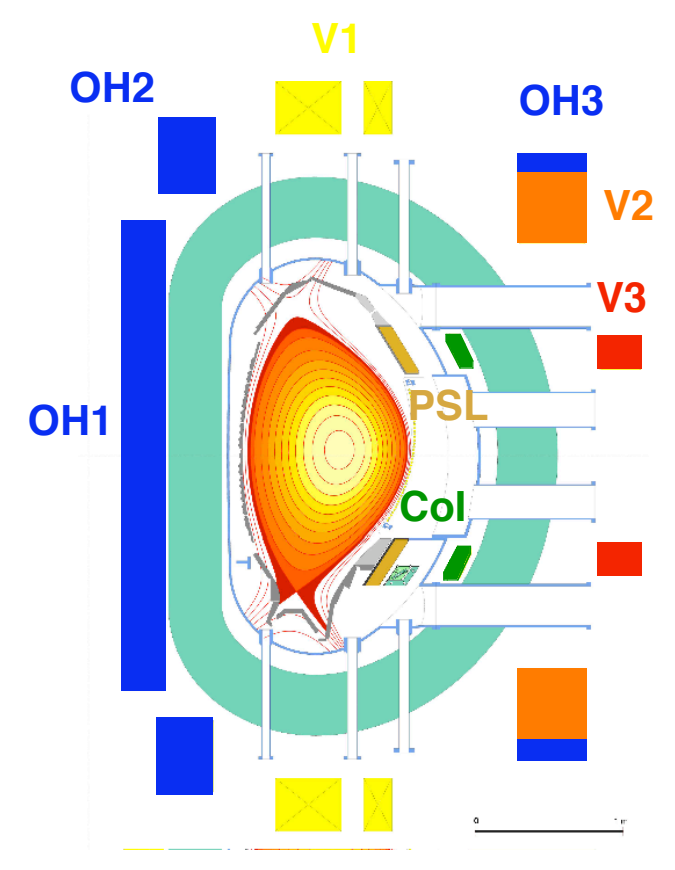

Figure 1: The cross section of the ASDEX Upgrade tokamak showing the ohmic heating coils (OH1, OH2 and $\mathrm{OH} 3)$, vertical field coils (V1, V2, and V3), vertical stabilization control coils (CoI) and passive stabilization coils (PSL).

The matrix equation relating the current in the 11 poloidal field coils, 


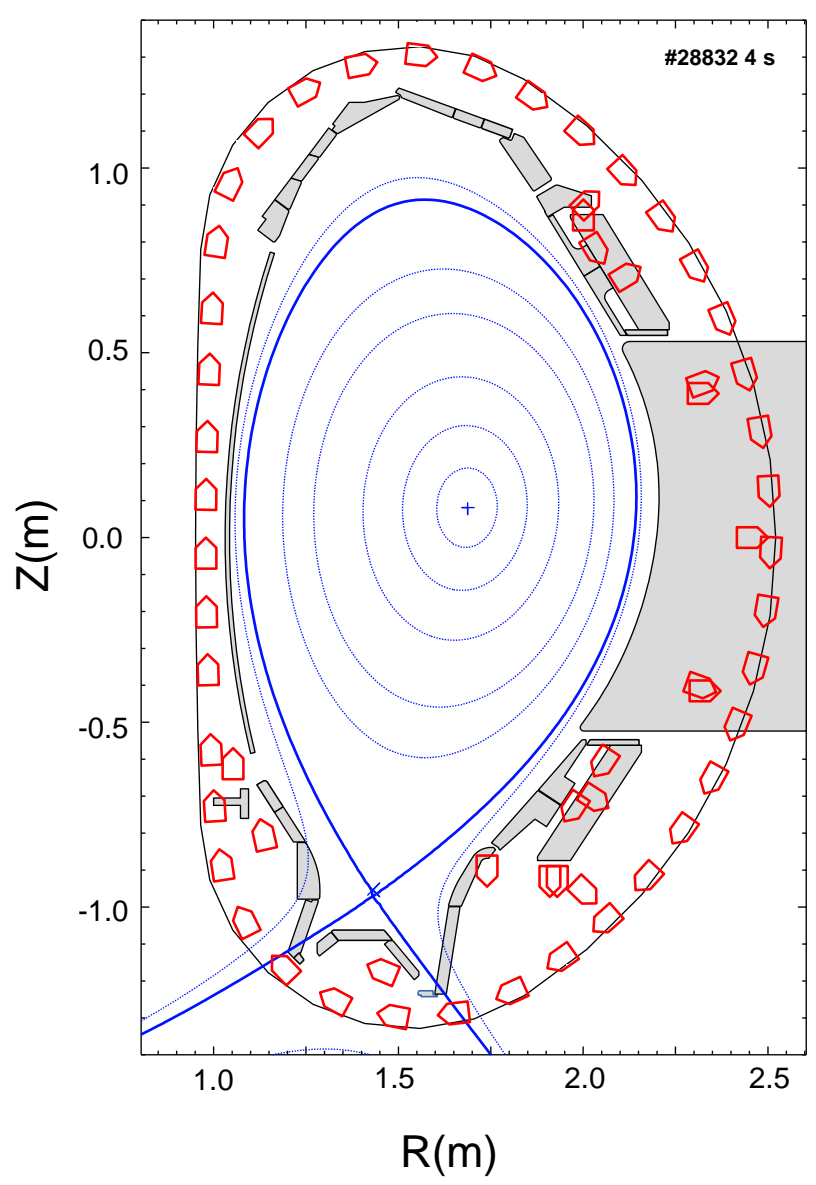

Figure 2: An overview of the position and orientation of the magnetic probes in ASDEX Upgrade. The flux surfaces of the magnetic equilibrium (dotted lines ) and the separatrix ( solid line ) are shown. 


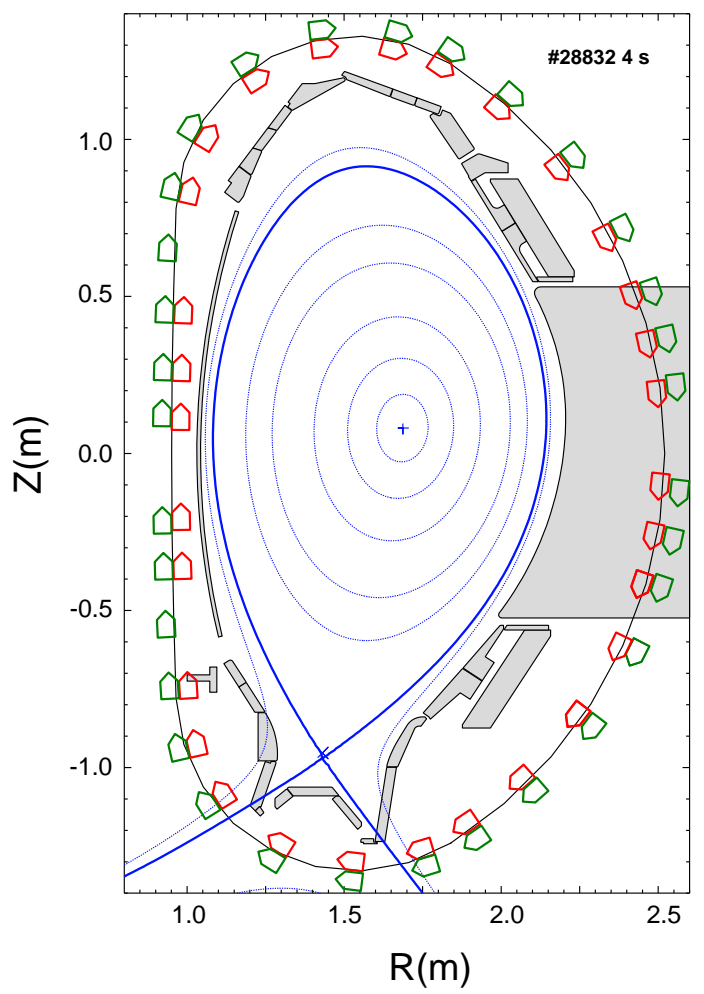

Figure 3: An overview of the position and orientation of the magnetic probes used for monitoring vessel currents in ASDEX Upgrade. The magnetic probes measure the poloidal field component inside (red) and outside (green) the vacuum vessel. 


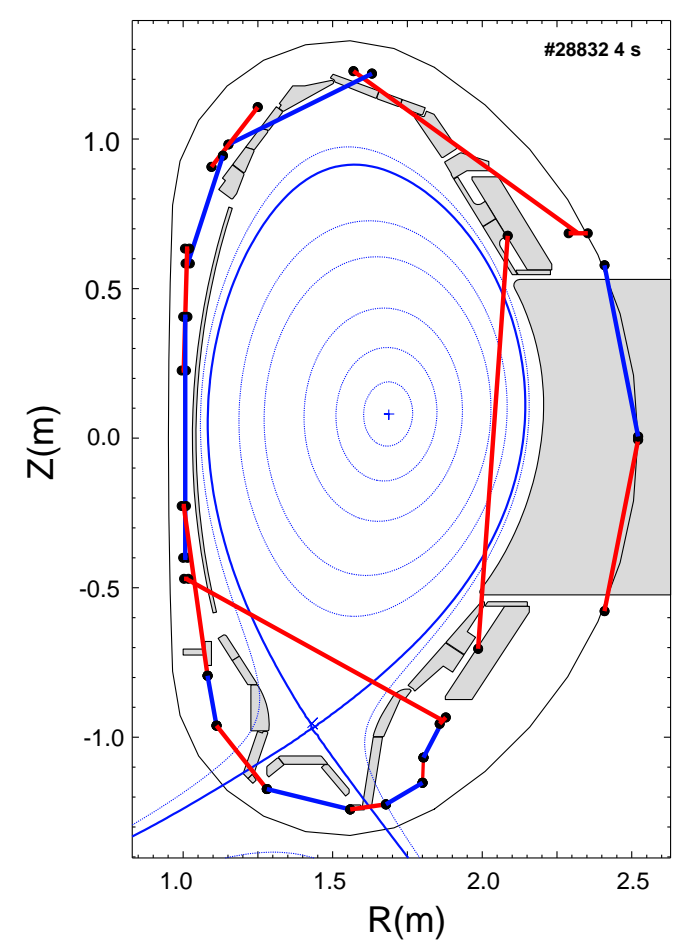

Figure 4: An overview of the position and orientation of the flux difference loops in ASDEX Upgrade. 
$\mathrm{I}_{j}$, the measurements of magnetic probe or the flux loop differences for each current, $m_{i j}$, can be expressed as :

$$
m_{i j}=M_{i j} I_{j}
$$

where $M_{i j}$ is the mutual inductance of the poloidal field coils and magnetic probes or flux loop differences. The $M_{i j}$ can be derived from the known position of the magnetic probe or flux loop and the coordinates of the poloidal field coil using the Greens function for the magnetic field generated by a current hoop as given in Eqns. 1, 2 and 3. Allowing for systematic calibration factor errors one can introduce scalings $g(i)$ for the probe measurements and $\mathrm{G}(\mathrm{j})$ for the Rogowski coil current measurements :

$$
g(i) m_{i j}^{*}=M_{i j} I_{j}^{*} G(j)
$$

where $\mathrm{m}_{i j}^{*}$ and $\mathrm{I}_{j}^{*}$ are the ideal best measurements of the magnetic probes and currents. The currents in each poloidal field coil were also measured with an accuracy of $0.1 \%$ with a Hitec STACC-HC zero flux system [24]. This allows the Rogowski current measurements to be controlled for accuracy once a year in dedicated test shots. The error in the nominal position of the poloidal field coils as $\Delta R_{j}$ and $\Delta Z_{j}$ and the variation in position and angle of the magnetic probes as $\Delta R_{i}, \Delta Z_{i}$ and $\Delta \theta_{i}$ are also possible variables for optimization. In this case the ideal mutual inductance, $\mathrm{M}_{i j}^{*}$, can be expressed as :

$$
M_{i j}^{*}=M_{i j}+\frac{\partial M_{i j}}{\partial R} \Delta R_{i}+\frac{\partial M_{i j}}{\partial a} \Delta R_{j}+\frac{\partial M_{i j}}{\partial Z}\left(\Delta Z_{i}+\Delta Z_{j}\right)+\frac{\partial M_{i j}}{\partial \theta} \Delta \theta_{i}
$$

The first step of the optimization process is concerned solely with finding the set of gains $g_{i}$ and $G_{j}$ to minimize the root mean square error of the difference between the predicted value and measured value of magnetic probes and flux loops in response to the excitation of 11 different poloidal field coils. In the second step, a steepest descent algorithm is used to optimize the 
positions of the flux loops. A factor of 4 reduction in the mean square error could be achieved. A comparison of the differences in measured and predicted values of 40 magnetic probes and 18 flux loops is shown for the case without ( Fig. 5 ) and with the optimization steps described ( Fig. 6 ). Allowing variations in the poloidal field coil positions reduced the root mean square error by a further $10 \%$. Position shifts of the order up to $2 \mathrm{~cm}$ in the radial and vertical direction were required to achieve this fit. Probe gains were adjusted in the range from 0.97 to 1.03 . In the final stage the radial position, vertical position and angle of the 40 magnetic probes were allowed to vary and this led to a further reduction in the root mean square error of $20 \%$ ( Fig. 7 ). These preliminary investigations were carried out with 100 iterations of the optimization algorithm. In the final analysis with 600 iterations of the optimization algorithm, a root mean square error of $0.7 \mathrm{mT}$ could be achieved for the difference between the measured and predicted values.

\section{Real-time Grad-Shafranov solver}

The magnetic equilibrium for a tokamak is described by the Grad-Shafranov equation :

$$
\begin{gathered}
\frac{\partial^{2} \psi}{\partial R^{2}}-\frac{1}{R} \frac{\partial \psi}{\partial R}+\frac{\partial^{2} \psi}{\partial Z^{2}}=-\mu_{0} R j_{\phi}(R, Z), \\
j_{\phi}(R, Z)=R \frac{\partial p}{\partial R}+\mu_{0} \frac{F}{R} \frac{\partial F}{\partial R}
\end{gathered}
$$

where $\psi$ is the poloidal flux per steradian, $j_{\phi}$ is the current density, and $R, Z$ and $\phi$ are the cylindrical coordinates. The plasma pressure, $p$, and poloidal plasma currents, $F=R b_{\phi} / \mu_{o}$, are functions of $\psi$. This problem is commonly solved by a cyclic reduction algorithm [17, 25, 26, 27]. A magnetic equilibrium for discharges with plasma current is reconstructed on a $33 \times 65$ grid using 40 magnetic probes and 18 flux loop difference signals. The right hand side current density term is calculated by a weighted least squares fit to the measurements which yields coefficients for the basis current density 


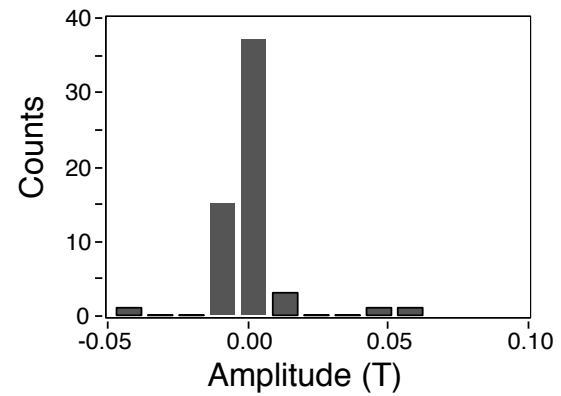

Figure 5: A histogram of the differences between the predicted and measured values of 40 magnetic probes and 18 flux loops without optimization.

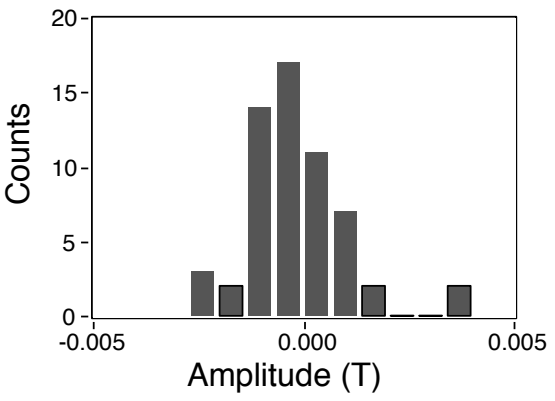

Figure 6: A histogram of the differences between the predicted and measured values of 40 magnetic probes and 18 flux loops with optimization of the flux loop position, measurement gains and calibration factor of the poloidal field current measurements.

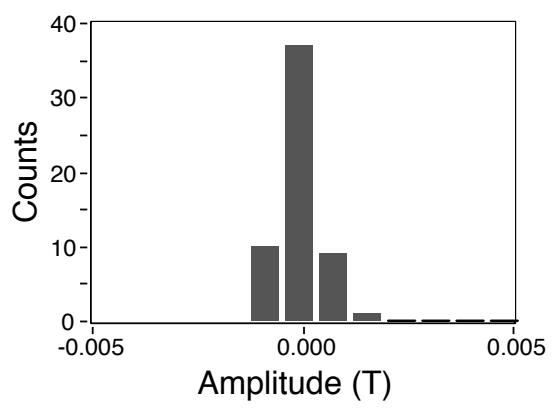

Figure 7: A histogram of the differences between the predicted and measured values of 40 magnetic probes and 18 flux loops with optimization of the flux loop position, measurement gains and calibration factor of the poloidal field current measurements, positions and angles of the magnetic probes and positions of the poloidal field coils. 
profiles [25, 26, 28]. Three basis current density profiles were chosen in the first round of development and found to adequately fit the experimental magnetic probe and flux loop measurements [23]. A fast, magnetics-free flux surface estimation and $q$-profile reconstruction algorithm for feedback control of plasma profiles also has been developed but has not yet been applied to NTM stabilization experiments [29].

A spectral-based algorithm to solve the Grad-Shafranov equation in an unbounded domain has been developed. This algorithm adapted a method commonly used to solve the Poisson equation in cylindrical coordinates. The use of discrete sine transforms (DST) along the $Z$-axis and a tridiagonal solver $[30,31,32]$ is an alternative to the cyclic reduction algorithm to solve the Grad-Shafranov equation [17].

\subsection{Spectral method}

A uniform mesh with constant spacing $d R$ and $d Z$ in the $R$ and $Z$ directions is assumed. The grid points are labeled from 0 to $N Z-1$ and 0 to $N R-1$, where $N Z$ is the number of grid points in the $Z$ direction, and $N R$ is the number of points in the $R$ direction. The five point difference equation with index $i$ in the $R$ direction and index $j$ in the $Z$ direction is :

$\frac{\psi_{i+1, j}-2 \psi_{i, j}+\psi_{i-1, j}}{d R^{2}}-\frac{1}{R_{i}} \frac{\psi_{i+1, j}-\psi_{i-1, j}}{2 d R}+\frac{\psi_{i, j+1}-2 \psi_{i, j}+\psi_{i, j-1}}{d Z^{2}}=-\mu_{o} R_{i} \mathbf{j}_{i, j}$

Introducing the discrete sine transform of $\psi$ and $\mathbf{j}$ :

$$
\begin{aligned}
\phi_{i, k} & =\sum_{j=1}^{N Z-2} \psi_{i, j} \sin \left(\frac{\pi j k}{N Z-1}\right) \\
J_{i, k} & =\sum_{j=1}^{N Z-2} \mathbf{j}_{i, j} \sin \left(\frac{\pi j k}{N Z-1}\right)
\end{aligned}
$$


leads to the tridiagonal matrix equations :

$$
\beta_{i} \phi_{i+1, k}-\alpha_{k} \phi_{i, k}+\gamma_{i} \phi_{i-1, k}=-\mu_{0} R_{i} d R^{2} J_{i, k}
$$

where $\beta_{i}=1-d R /\left(2 R_{i}\right), \gamma_{i}=1+d R /\left(2 R_{i}\right), S=d R / d Z$ and

$\alpha_{k}=2+4 S^{2} \sin ^{2}\left(\frac{\pi k}{2(N Z-1)}\right)$.

\subsection{Tridiagonal solver}

The tridiagonal matrix equation is solved with a tridiagonal solver using an LU decomposition algorithm ( where the tridiagonal matrix is expressed as the product of a lower triangular matrix and an upper triangular matrix). These two bidiagonal matrices are subsequently used in the iterative procedure to solve the tridiagonal equations. By using LU decomposition, operations are reduced by a factor of 2 compared to the direct solver algorithm [33].

\subsection{Unbounded domain}

The solver for the Grad-Shafranov equation in an unbounded domain is composed of two fast solver steps [17]. The new algorithm introduces novel ideas in the spectral method at each step to reduce the computing time dramatically [34].

The first step of the solver uses zero as the condition for all grid boundaries with a right hand side current distribution on the flux surfaces from the previous iteration given by the weighted least squares fit to the magnetic probe and flux loop measurements. In this step, it is only necessary to compute $\psi$ at points neighbouring the grid boundary and a reduced inverse DST can be performed to calculate these values. The columns of $\psi$ inside the boundary edge are :

$$
\psi_{i, k}=\frac{2}{N Z-1} \sum_{j=1}^{N Z-2} \phi_{i, j} \sin \left(\frac{\pi j k}{N Z-1}\right)
$$


where $i=1$ and $N R-2$, and the rows inside the boundary edge can be calculated in a similar fashion with $k=1$ and $N Z-2$. All these four edges can be computed using matrix-vector multiplication. This avoids the unnecessary computations performed by a traditional inverse DST operation applied to the entire grid. The gradients in $\psi$ normal to the grid boundary, $(\partial \psi / \partial n)_{\text {boundary }}$, are the inputs required for the next solver step. These are the shielding currents that are necessary to force the zero boundary condition of the first solver step. They are used to calculate the Green's functions for $\psi$ generated by a current hoop of radius, $a$, carrying current, $I$, for each grid point with radial coordinate, $R$, and a vertical distance, $Z$, on the boundary. The self-inductance of a current element on the boundary is given by :

$$
\psi=\frac{\mu_{o} I}{2 \pi} \Delta l\left(1+\ln \frac{\Delta l}{16 R}\right)\left(\frac{\partial \psi}{\partial n}\right)_{\text {boundary }}
$$

where $\Delta \mathrm{l}=d R$ for elements on the boundary along a radial axis or $\Delta \mathrm{l}=$ $d Z$ for elements on the boundary along the $Z$-axis. The calculation of the resulting $\psi$ on the boundary is performed as a matrix multiplication with pre-calculated coefficients times the vector of shielding currents.

The second step of the solver is carried out with boundary conditions from the first solver step but without current source terms on the right hand side of the Grad-Shafranov equation. Because only the first and last elements in the vertical direction are nonzero, it is possible to use an optimized DST to reduce the computation effort. The faster DST is carried out by evaluating only the sum of the two non-zero terms :

$$
\begin{aligned}
D_{i j} & =-\frac{\psi_{i, 1} \sin \left(\frac{\pi j}{N Z-1}\right)+\psi_{i, N Z-2} \sin \left(\frac{\pi j(N Z-2)}{N Z-1}\right)}{d Z^{2}} \\
& =-\frac{\psi_{i, 1}-(-1)^{j} \psi_{i, N Z-2}}{d Z^{2}} \sin \left(\frac{\pi j}{N Z-1}\right)
\end{aligned}
$$

The DST of the boundary conditions at the inner and outer radial positions are added to the first and last columns. The tridiagonal solver is 
applied to this result and is added to the result from the first solver step. The solution of the Grad-Shafranov equation is then calculated by an inverse DST. Under equivalent boundary conditions, an implementation based on the cyclic reduction algorithm computes all elements on the grid in both solver steps. The Grad-Shafranov solver algorithm described here achieves a significant performance improvement in comparison to cyclic reduction by emp4 $\times$ 4loying two optimized DST implementations. The first implementation exploits the ability to avoid unnecessary calculations. The second implementation exploits the fact that the right hand side term is zero except at the boundary. This reduces the number of operations needed to solve the Grad-Shafranov equation.

The $\psi$ generated by the external poloidal field coils and passive stabilizing loop on the grid is also realized as a matrix-vector multiplication using factors calculated with Eq. 1. The poloidal field coils and passive stabilizing loop are simulated as a finite number of filaments, with each filament carrying an applicable number of turns.

In experiments on the ASDEX Upgrade tokamak, the use of magnetic perturbation coils used for ELM mitigation experiments [35] requires that the probe measurements be corrected for contributions due to the DC excitation of these coils. The mutual inductances between each of the 16 installed perturbation coils and the magnetic probe measurements are recorded in vacuum field calibration discharges. These corrections can be carried out in real-time.

A parallelization of the solution of the Poisson equation using fast Fourier transforms was proposed in a number of publications [36, 37, 38]. Recently, an application using such a parallel algorithm for the solver of the GradShafranov equation has been successfully implemented. A cycle time of $0.076 \mathrm{~ms}$ on a $33 \times 65$ grid using a single core and $0.040 \mathrm{~ms}$ on 4 cores of a $3.2 \mathrm{GHz}$ Xeon W5580 CPU could be achieved [31, 39]. The solver presented here is a single thread solution only and the DST is performed as a 
matrix multiplication to allow flexible choice of the grid size. On a $33 \times 65$ grid, the solver requires $0.09 \mathrm{~ms}$. The grid extends from $0.91 \mathrm{~m}$ to $2.27 \mathrm{~m}$ in the radial direction and $-1,36 \mathrm{~m}$ to $1.36 \mathrm{~m}$ in the vertical direction. Therefore the grid points are separated by $4.25 \mathrm{~cm}$ in the radial direction and vertical direction. An option for using a 49x97 grid has been tested. The grid point separation in this case will be $2.84 \mathrm{~cm}$ in the radial and vertical direction.

The magnetic axis was located as the maxima of a second order polynomial surface fit on a $4 \times 4$ grid in the vicinity of the maximum value of $\psi$ on the grid. The limiter value of $\psi$ is the maximum value of the linearly interpolated $\psi$ value on a set of coordinates describing the limiter. The location of the grid coordinates closest to the $X$-point is found by looking for the row minimum and column maximum on the grid on a subset of the $\psi$ matrix where the $X$-point normally exists. The coordinates of the upper and lower X-points are then calculated from the saddle point of a second order polynomial surface fit to a $3 \times 3$ grid in the vicinity of this location.

Plasma shape control in rtEFIT is carried out by the isoflux algorithm. In this scheme, the value of the current in the poloidal field coils is varied so that the value of $\psi$ at a number of control points is held constant [25]. In this case, the differences in $\psi$ at the control points, $\delta \psi_{i}$, are related simply to the necessary change in currents, $\delta I_{j}$, in the control coils by the matrix equation :

$$
\delta \psi_{i}=C_{i j} \delta I_{j}
$$

where $C_{i j}$ is the mutual inductance of the poloidal field coils and the position of the control point given by Eq. 1. This matrix equation is solved for the right hand side with a least squares algorithm. In ASDEX Upgrade, the currents required in 6 shaping coils for 4 control points have been successfully been implemented. Interpolation of the pre-calculated response of the magnetic field generated by the poloidal field coils on the grid points of the solver was necessary to solve Eq. 16 in real-time. 


\section{Contour integrals}

The safety factor, $q\left(\psi_{N}\right)$, along the contour of constant normalized poloidal flux, $\psi_{N}$, is [40] :

$$
q\left(\psi_{N}\right)=\frac{B_{o} R_{o}}{2 \pi} \oint_{C} \frac{1}{R^{2} b_{p o l}} d s
$$

where $B_{o}$ is the toroidal magnetic field on the torus axis at position $R_{o}, b_{\text {pol }}$ is the poloidal magnetic field and $\psi_{N}$ is defined as [25] :

$$
\psi_{N}=\frac{\psi-\psi_{\text {axis }}}{\psi_{\text {boundary }}-\psi_{\text {axis }}}
$$

where $\psi_{\text {boundary }}$ and $\psi_{\text {axis }}$ are the values of poloidal magnetic flux at the boundary and axis respectively.

The line elements of the contours of constant $\psi$ are returned by a contouring subroutine. The $b_{r}$ and $b_{z}$ components of the poloidal magnetic field at the midpoints of the line elements forming the contour are evaluated by four point interpolation of the gradients of $\psi$ on the grid.

The first step of locating the normalized radius of the $q$ surface at rational surfaces with values of $m / n=4 / 3,3 / 2$ and $2 / 1$ from the poloidal flux matrix is the evaluation of contour integrals for $q$ at ten values of normalized poloidal flux. These contour integrals are carried out in five parallel instances of the subroutine. The normalized radius of the chosen rational surface is then found by spline interpolation of the normalized poloidal flux for the contour integrals where the normalized radius, $\rho_{N}$, is defined as :

$$
\rho_{N}=\sqrt{\psi_{N}}
$$

The contour integrals and the spline interpolation are performed in $0.30 \mathrm{~ms}$. The line integrated phase shift of five chords of a DCN laser interferometer are processed using the flux surfaces and the lines of sight to calculate a set of coefficients describing Abel inverted electron density profile in real-time 
[14]. From the flux matrix and the lines of sight of the ECE diagnostic, the location of the normalized radius of each frequency of measurement is calculated in real-time. The three values of normalized radius of the rational surfaces, the set of coefficients representing the electron density profile, the normalized radius of each ECE channel and the poloidal flux matrix are communicated in real-time for performing the TORBEAM microwave refraction beam tracing and for feedback control of the NTM.

The Shafranov integrals, $S_{1}$ and $S_{2}$ are contour integrals on the last closed flux surface $[27,40,41,42,43,44]$ :

$$
\begin{aligned}
S_{1} & =\frac{\pi}{V \bar{B}_{p a}^{2}} \iint R b_{p o l}^{2}\left(\left(R-R_{o}\right) \bar{e}_{R}+Z \bar{e}_{Z}\right) \cdot \bar{n} d s \\
& =\frac{\pi s^{2}}{V\left(\mu_{o} I_{p}\right)^{2}} \oint_{C} R b_{p o l}^{2}\left(\left(R-R_{o}\right) \bar{e}_{R}+Z \bar{e}_{Z}\right) \cdot \bar{n} d s \\
S_{2} & =\frac{\pi}{V \bar{B}_{p a}^{2}} \iint R b_{p o l}^{2} R_{o} \bar{e}_{R} \cdot \bar{n} d s \\
& =\frac{\pi s^{2}}{V\left(\mu_{o} I_{p}\right)^{2}} \oint_{C} R b_{p o l}^{2} R_{o} \bar{e}_{R} \cdot \bar{n} d s
\end{aligned}
$$

where $\bar{n}$ is the vector perpendicular to the line element of the contour, $s$, is the distance around the contour, $\bar{B}_{p a}=\left(\mu_{o} I_{p}\right) / s$ is the mean poloidal field around the contour and $V$ is the plasma volume. The poloidal beta, $\beta_{p}$, and the plasma inductance, $l_{i}$, are calculated from these integrals:

$$
\begin{aligned}
l_{i} & =\frac{1}{V \bar{B}_{p a}^{2}} \int b_{p o l}^{2} d V \\
& =\frac{2 \pi s^{2}}{V\left(\mu_{o} I_{p}\right)^{2}} \int R b_{p o l}^{2} d R d Z \\
\beta_{p} & =0.5 S_{1}+\left(1-0.5\left(1-\frac{R_{c}}{R_{o}}\right)\right) S_{2}-0.5 l_{i}
\end{aligned}
$$

where $R_{c}$ is the radius of the magnetic axis. 


\section{MSE constraints}

A third loop executes a Grad-Shafranov solver that additionally fits ten spatially localized measurements from the Motional Stark Effect diagnostic [45]. The accuracy of the $q$ profile is improved by the measurements of the polarization angle, $\gamma_{m}$ which is related to the viewing geometry [46]:

$$
\tan \left(\gamma_{m}\right)=\frac{c 1 * b_{r}+c 2 * b_{\phi}+c 3 * b_{z}+c 4 * E_{r} / v \text { Beam }}{c 5 * b_{r}+c 6 * b_{\phi}+c 7 * b_{z}+c 8 * E_{r} / v \text { Beam }+c 9 * E_{z} / v \text { Beam }}
$$

where $c, \ldots, c 9$ are a set of coefficients for each channel relating the local components of electric field $\left(E_{r}\right.$ and $\left.E_{z}\right)$, the radial, vertical and toroidal components of magnetic field $\left(b_{r}, b_{z}\right.$ and $\left.b_{\phi}\right)$ and diagnostic beam velocity, vbeam [28, 47, 48]. The components of the poloidal magnetic field at the centre of the measurement volume are also evaluated by a matrix-vector multiplication using pre-calculated Green's functions. The toroidal component of the magnetic field, $\mathrm{b}_{\phi}$, is calculated from the definition of $F F^{\prime}$ :

$$
\left(R b_{\phi}\right)^{2}=\left(R_{o} B_{o}\right)^{2}+2 \mu_{o}^{2} \int_{\psi_{\text {boundary }}}^{\psi} F F^{\prime} d \psi
$$

where $F F^{\prime}$ are those terms of the current profile representing the poloidal current. The integrals of the current basis functions representing the $F F^{\prime}$ term are tabulated so that the expression can be evaluated in real-time.

In the mid-plane, $E_{z}$ can be neglected as the viewing angle of the MSE from the horizontal plane is small [48]. The value of $E_{r}$ can be either measured by a second view of another MSE system of the same volume or calculated from the force balance equation using charge exchange recombination spectroscopy measurements [46, 49] or reflectometry measurements [50]. On ASDEX Upgrade, real-time measurements of $E_{r}$ are not yet available. The only possibility to account for them would be to use $E_{r}$ radial profiles from previous similar discharges. This assumes that NTM stabilization does not 
introduce changes in the radial profiles that need to be accounted for in Eq. 24. It is known that neglecting the term in electric field leads to differences in the inferred value of the central safety factor, $q(0)$ [48].

The expression for the terms of the response matrix for each MSE channel is then :

$$
\begin{array}{r}
\left(c 1-c 5 \tan \left(\gamma_{m}\right)\right)\left(b_{r p}+b_{r c}\right)+\left(c 3-c 7 \tan \left(\gamma_{m}\right)\right)\left(b_{z p}+b_{z c}\right)= \\
-\left(c 2-c 6 \tan \left(\gamma_{m}\right)\right) b_{\phi}-\left(c 4-c 9 \tan \left(\gamma_{m}\right)\right) * E_{r} / v \text { Beam }
\end{array}
$$

The left hand side terms of the response matrix require the magnetic field components, $b_{r c}$ and $b_{z c}$ calculated for each MSE measurement volume and for each current basis function. The $b_{r c}$ and $b_{z c}$ are the components due to currents in the poloidal field coils for each MSE measurement volume and are transferred to the right hand side of the above equation. The ten additional constraints on the response matrix typically allows the number of fit coefficients to be raised from 4 to 6 [45]. A simulation of the MSE diagnostic measurements was created to allow the application to be tested. The expected $\tan \left(\gamma_{m}\right)$ for an equilibrium fitting the magnetic probes and flux loops was calculated and subsequently used to calculate an equilibrium using magnetics and the simulated MSE measurements. In Figs. 8 and 9, the simulated and fitted right hand side of the response matrix for each MSE measurement volume are plotted.

In the NTM stabilization experiments discussed below, MSE measurements were not routinely available for the real-time magnetic equilibria calculations. The MSE diagnostic neutral beam for the central electron density of the discharge was sufficiently attenuated to make the central MSE channel measurements unreliable. Routine operation covering a wider electron density range will necessitate increasing the diagnostic beam energy. 


\section{Data acquisition system}

A Supermicro X9DRL-3F motherboard, with two octal core Xeon E52687 3.1 GHz CPU's and LabVIEW RT 2011 RT SP1, is in routine operation for the calculation of real-time magnetic equilibria. A 4× PCIe VMIC 5565 PIORC reflective memory card and a NI PCIe 8362 interface card connects a PXI 1045 chassis for the data acquisition of 80 channels at a sample rate of $10 \mathrm{kHz}$ with 10 PXI 6143 cards. The reflective memory card transmits the $33 \times 65$ poloidal flux matrix value to the control system with less than $1 \mathrm{~ms}$ delay. For diagnostics not connected to the reflective memory network, a compressed flux matrix that fits into a UDP packet is available. The compression performs a two dimensional discrete cosine transform (DCT) and sends the 253 DCT coefficients to the remote real-time diagnostic for decompression of the flux matrix by an inverse DCT. A third party PCI card delivers 64 bit time stamps using a $100 \mathrm{MHz}$ clock and generates the $10 \mathrm{MHz}$ TTL pulses for clock synchronization of the data acquisition boards in a number of data acquisition systems. The clock synchronization and trigger pulses are delivered to the data acquisition boards by an internal bus.

A cycle time of $0.4 \mathrm{~ms}$ using the real-time Grad-Shafranov solver with 40 magnetic probe and 18 flux loop differences as constraints and fitting 4 coefficients using $4 \mathrm{CPU}$ cores was achieved. A cycle time of $0.6 \mathrm{~ms}$ using the real-time Grad-Shafranov solver when fitting 6 coefficients with the addition of a further 10 constraints from MSE measurements was achieved. In this case, parallel solvers with and without MSE constraints are executed. This allows the feedback control system the opportunity of immediately switching back to a flux matrix solution constrained only by magnetic probes and flux loop differences in the event that the MSE measurements are no longer possible. The operation of the MSE diagnostic depends on a particular neutral injection beam source and measurements are not possible if the source is not available at some time during the discharge. Simultaneously, a function parameterization algorithm is performed. The plasma current, its radial 
and vertical position and 95 other values of interest for plasma control are calculated [51].

A schematic diagram of the software (JANET - Just ANother Equilibrium reconstruction for Tokamaks ) is shown in Fig. 10. With parallel solvers, data acquisition and real-time communication to the control system, a cycle time of $1.5 \mathrm{~ms}$ could be maintained. Minimum jitter of the cycle times was achieved by assigning a dedicated CPU to the data acquisition loop. The achieved cycle time is therefore satisfactory for the real-time processing requirements of NTM stabilization experiments where the cycle time of the discharge control system is $1.3 \mathrm{~ms}$ [52]. The TORBEAM calculations typically require $20 \mathrm{~ms}$ and the MSE measurements were available every $10 \mathrm{~ms}$. A cycle time of $2 \mathrm{~ms}$ for the solvers with real-time communication to the control system every $4 \mathrm{~ms}$ was used in the NTM stabilization experiments presented. It should be noted that these benchmarks are for a single cycle iteration for the PDE solution. On DIII-D, it has been shown that when comparing real-time magnetic equilibria from rtEFIT with well converged solutions from offline EFIT calculations, that the small differences found for relatively steady state conditions are not relevant for practical discharge control [25].

\section{NTM stabilization experiments}

A NTM mode with $m / n=3 / 2$ was present in a 1 MA discharge with up to 12.5 MW NBI heating and 1.4 MW ECRH heating. The location in normalized radius of this mode can be inferred from temperature fluctuation measurements at the mode frequency of the NTM. The phase jump of the fluctuation is related to the change in phase of the temperature fluctuation around the NTM magnetic island $[2,5,53]$. These measurements combined with the magnetic equilibrium indicate that the NTM is located at a normalized radius of about 0.6. The basis current profiles for the Grad-Shafranov solver are chosen so that the predicted normalized radius is sufficiently ac- 
curate to perform NTM stabilization experiments. In Fig. 11, a contour plot of ECE temperature as a function of normalized radius in the presence of a NTM is shown. In Fig. 12, is the TORBEAM calculation for the two gyrotrons used in these experiments, with the mirror launcher for the off-axis gyrotron used for NTM stabilization tilted at a toroidal angle of $-4.82^{\circ}$ for ECCD.

The feedback control of the mirror for NTM stabilization experiments is based on the difference of the TORBEAM calculated normalized deposition radius, $\rho_{E C R H}$, and the calculated normalized radius of the $m / n$ rational surface from the real-time equilibrium, $\rho_{N T M}$. The change in position of the mirror actuator, $\Delta x_{p o l}$ was calculated from the relation :

$$
\Delta x_{p o l}=\frac{\partial x_{p o l}}{\partial \rho_{E C R H}} \Delta \rho
$$

where $\Delta \rho=\rho_{E C R H}-\rho_{N T M}$. The value of $\frac{\partial x_{p o l}}{\partial \rho_{E C R H}}$ is calculated from three parallel calculations of TORBEAM with small differences in $x_{p o l}$. The value of $\Delta x_{p o l}$ is the input to a PI controller with proportional gain, $K_{p}=0.2$, and integral gain, $K_{i}=6.0$. The output of this controller is passed as a command to the proprietary motor controller to drive the mirrors. The full loop has a settling time of approximately $300 \mathrm{~ms}$ for large step inputs with overshoot smaller than the measurement noise and zero steady state error. Simulations of feedback control have been carried out to optimize the mirror controller [54].

Shown in Fig. 13, is the level of agreement between the normalized radius measured by temperature fluctuations at the NTM frequency and the predicted normalized radius of the $m / n=3 / 2$ rational surface by the real-time Grad-Shafranov solver. In this discharge, the mirror angle was scanned to vary ECCD power deposition as a function of the normalized radius. From the magnetic fluctuation amplitude of the $m / n=3 / 2$ mode, the NTM is present after $2.4 \mathrm{~s}$ while at $5.1 \mathrm{~s}$ a small dip corresponding to partial ECCD 
stabilization is indicated. From this dip it is inferred that the NTM is positioned at a normalized radius of 0.57 , while the temperature fluctuations yield a value of 0.58 and from the magnetic equilibrium a value of 0.60 is predicted. If the current profile were sufficiently well determined by the Grad-Shafranov solver, it would be expected that all three measurements would be in exact agreement. It is known that owing to the lack of constraints in the plasma centre, the internal current density profile and $q$ profile inferred from external magnetic measurements alone are not able to be accurately determined [55]. In these experiments, a pre-programmed mirror angle offset is required to perfectly align the ECCD and mode location from the Grad-Shafranov solver. The inclusion of MSE measurements to more accurately determine the current profile in the plasma centre in future experiments, should remove the need for using a pre-programmed offset in mirror angle.

It also has been observed that the magnetic island may not be symmetric around a flux surface [56]. This implies that the rational flux surface and the optimal point of normalized radius for ECCD deposition in the centre of the magnetic island may not be aligned. This would suggest that a small offset may need to be introduced for pre-emptive stabilization experiments using the normalized radius of the rational flux surface calculated by the GradShafranov solver. Modeling has shown that the deformation of the magnetic island caused by the viscous drag of the sheared flow is consistent with electron temperature fluctuation measurements on DIII-D [57]. An additional point for understanding the need for a pre-programmed offset could be related to the uncertainty in the absolute value of the toroidal magnetic field. The evaluation of modulated ECRH power experiments and the measured phase and amplitude response of ECE channels shows that the measured deposition radius is accurate to within $\pm 0.5 \%$ of the nominal toroidal field inferred from a highly accurate toroidal field coil current measurement $( \pm 0.1 \%)$. Such a systematic error would lead to changes in the calculated normalized radius of the ECCD deposition and the ECE channel location. 
In Fig. 14, the mirror is aimed by feedback control to place the ECCD deposition at the normalized radius of the mode predicted by the real-time Grad-Shafranov solver plus normalized radius offset. The mirror angle also includes compensation for microwave beam refraction in the presence of electron density gradients calculated in real-time using the TORBEAM code. The amplitude of the magnetic fluctuations at the NTM mode frequency are reduced and the NTM is stabilized, even in the extended period of maximum NBI heating. The NTM cannot be localized as the temperature fluctuation amplitude of the NTM has been greatly reduced. Consequently, the normalized radius of the NTM measured by temperature fluctuations displays a large scatter. In this discharge only $700 \mathrm{~kW}$ of ECCD power was required to stabilize the NTM. In similar discharges where the NTM was present, it was found that the NTM could only be partially stabilized with double the power. An ECRH power of $900 \mathrm{~kW}$ was used in pre-emptive NTM stabilization experiments on DIII-D in discharges with $7 \mathrm{MW}$ of neutral beam heating power [13].

In Fig. 15, feedback control of the mirror is carried out until $3.1 \mathrm{~s}$. Up until this time, the NTM mode partially grows for a short time again as indicated by an increase in the magnetic fluctuations at the NTM mode frequency. The NTM mode is then suppressed once more before feedback control is stopped. The NTM begins to grow again after the mirror is purposefully moved away from the predicted stabilizing position. The growth of the NTM during pre-emptive stabilization has been previously observed and the cause of this phenomenon is thought to be associated with changes in the magnetic equilibrium. Evidence for this can be inferred from the increase in the plasma stored energy, $W_{m h d}$, up until the time that the mode appeared. In this discharge the $\beta_{p}$ is slightly larger than in the previously considered discharge. This indicates that the increase in Shafranov shift associated with the increased $\beta_{p}$ during NTM stabilization and the shift in the radial location of the NTM is not satisfactorily delivered by the real-time 
equilibrium calculations. The location in normalized radius of the mode inferred from the temperature fluctuations at this time also indicate that the mode moved inwards, so that it is possible that the mode was destabilized by ECCD deposition outside of the rational $q$ surface. The most challenging unsolved task of pre-emptive stabilization will be coping with changes in the magnetic equilibrium due to increases in the plasma pressure as the result of NTM stabilization and the probable excitation of NTM modes at other rational surfaces. It is concluded that it is absolutely necessary to include MSE constraints to reconstruct the magnetic equilibrium in the plasma centre with sufficient accuracy for pre-emptive NTM stabilization to be suitable for routine operation. Nevertheless, pre-emptive stabilization has been achieved using the real-time estimated rational flux surface plus an offset. The data acquisition system was running with parallel solvers, with the second solver including the calculated MSE constraints as an input. Therefore, it also has been demonstrated that the data acquisition system would have simultaneously delivered the normalized radius of the NTM with a magnetic equilibrium reconstruction including MSE constraints.

The MHD signatures of these discharges are now compared. In Fig. 16, where the mirror for ECCD deposition was scanned across the normalized radius, in the period with $12.5 \mathrm{MW}$ NBI heating power a $3 / 2 \mathrm{NTM}$ mode at $33 \mathrm{kHz}$ can be identified and in the period with $10 \mathrm{MW}$ NBI heating power a $3 / 2$ NTM mode at $25 \mathrm{kHz}$ can be identified. In Fig. 17, for the discharge where the mirror for ECCD deposition was feedback controlled for pre-emptive stabilization, in the period with 12.5 MW NBI heating power a $3 / 2$ NTM mode at $25 \mathrm{kHz}$ and a $4 / 3$ NTM mode at $45 \mathrm{kHz}$ can be identified and in the period with $10 \mathrm{MW}$ NBI heating power only a 4/3 NTM mode at $38 \mathrm{kHz}$ can be identified. It seems that the NTM mode was only partially stabilized for the period with higher NBI power, while the NTM mode was fully suppressed for the period with lower NBI power. In Fig. 18, the offline magnetic equilibrium and real-time equilibrium are compared. The number 
and form of the current basis functions are not the same, so that perfect agreement cannot be expected. In Fig. 19, for the discharge where the mirror for ECCD deposition was feedback controlled up to $3.1 \mathrm{~s}$, in the period with 12.5 MW NBI heating power a $3 / 2$ NTM mode at $33 \mathrm{kHz}$ can be identified and as $\beta_{p}$ is reduced the mode frequency is reduced to $30 \mathrm{kHz}$. In the period with 10 MW NBI heating power only a $3 / 2$ NTM mode at $30 \mathrm{kHz}$ can be identified. This mode frequency reduced to $25 \mathrm{kHz}$ as the mirror was moved away from the normalized radius predicted for stabilization and the mode amplitude grew.

\section{Conclusion}

A real-time Grad-Shafranov solver based on a discrete sine transformation of the difference equation rather than cyclic reduction has been realized. The resulting tridiagonal equations are solved with a specially developed subroutine based on LU factorization. This tridiagonal solver reduces the number of operations with respect to the iterative direct solver by pre-calculating the reciprocal of the diagonal elements. A reduced inverse DST is required in the first solver step as only the relevant terms for those neighbours of the grid boundary need be calculated. A simplified DST can be used for the second solver step where only the first and last elements are non-zero. In this way the full inverse DST of the first solver step is omitted and the DST of the second solver step without current source terms can be calculated with a smaller number of operations. Contour integrals on flux surfaces of the poloidal flux matrix allows real-time evaluation of the normalized radius of rational $q$ surfaces, $\beta_{p}$ and $l_{i}$. The magnetic probe measurements are accurately calibrated by an optimization process to find the values of probe position and orientation, poloidal field coil position and integrator gains that minimize the error between the calculated and measured probe responses to a current pulse in each of the poloidal field coils. A cycle time of $0.6 \mathrm{~ms}$ for calculating two tokamak equilibria in parallel using four current basis functions 
with magnetic probe measurements as constraints only and using six current basis functions with magnetic probe and MSE measurements as constraints has been achieved. For NTM stabilization experiments, with data acquisition and real-time communication, a cycle time of $2 \mathrm{~ms}$ was used. This cycle time satisfies the real-time processing requirements for NTM stabilization experiments on ASDEX Upgrade.

The real-time magnetic equilibrium reconstructions for NTM stabilization experiments were constrained by the magnetic probe and flux loop difference measurements only, as robust real-time MSE measurements were not available. Feedback control of a mirror launcher for pre-emptive NTM stabilization experiments using real-time magnetic equilibria could be demonstrated. A pre-programmed launcher mirror angle offset is required to perfectly align the ECCD and NTM location in these experiments as the normalized radius predicted by the equilibrium lies slightly outside the measured location. However, in a subsequent discharge the NTM was initially stabilized but then grew for a short time during the period of ECCD deposition. This was due to a change in the radial location of the mode that was not able to be recovered by equilibrium constraints using magnetic probe measurements only. It is concluded that additional MSE constraints for equilibrium reconstruction are essential to more accurately determine the spatial location of the rational $q$ surface and make pre-emptive NTM stabilization a robust tool for improving plasma performance.

\section{Acknowledgements}

Many thanks to R. Cole, M. Fitzek and K. Lüddecke for their development of the real-time communication software and time stamping hardware. The interesting discussions with members of the real-time magnetic equilibrium reconstruction team at DIII-D (J.Ferron, H.St.John and E.Straits) concerning the details of rtEFIT were greatly appreciated. The integration of this diagnostic into the control and data acquisition system of ASDEX 
Upgrade was made possible by the assistance of the data acquisition group ( K. Behler, A. Buhler, H. Blank, S. Martinov and R. Merkel) and the control group (G. Neu, D. Zasche and T. Zehetbauer). 


\section{References}

[1] M. Maraschek, G. Gantenbein, T.P. Goodman, S. Günter, D.F. Howell, F. Leuterer, et al., Active control of MHD instabilities by ECCD in ASDEX Upgrade, Nucl. Fusion 45 , 2005, 1369.

[2] M. Reich, A. Bock, K. Behler, L. Giannone, M. Maraschek, E. Poli, et al., Europhysics Conference Abstracts (Proc. of the 38th EPS Conference on Plasma Physics, Strasbourg, France) 2011, http://ocs.ciemat.es/EPS2011PAP/pdf/P5.102.pdf.

[3] G. Gantenbein, H. Zohm, G. Giruzzi, S. Günter, F. Leuterer, M. Maraschek, et al., Complete suppression of neoclassical tearing modes with current drive at the Electron-Cyclotron-Resonance frequency in ASDEX Upgrade Tokamak, Phys. Rev. Lett., 85, 2000, 1242.

[4] K. Nagasaki, A. Isayama, N. Hayashi, T. Ozeki, M. Takechi, M. Oyama, et al., Stabilization of neoclassical tearing mode by ECCD and its evolution simulation on JT-60U tokamak, Nucl. Fusion, 45, 2005, 1608.

[5] R. Prater, R.J. La Haye, T.C. Luce, C.C. Petty, E.J. Strait, J.R. Ferron, et al., Stabilization and prevention of the $2 / 1$ neoclassical tearing mode for improved performance in DIII-D, Nucl. Fusion, 45, 2007, 371.

[6] A. Imsayama, G. Matsunaga, T. Kobayashi, S. Moriyama, N. Oyama, Y. Sakamoto, et al., Neoclassical tearing mode control using electron cyclotron current drive and magnetic island evolution in JT-60U, Nucl. Fusion, 49, 2009, 055006.

[7] D.A. Humphreys, J. Ferron, R.J. La Haye, T.C. Luce, C.C. Petty, R. Prater, et al., Active control for stabilization of neoclassical tearing modes, Phys. Plasmas, 13, 2006, 056113. 
[8] R. Prater, R.J. La Haye, J. Lohr, T.C. Luce, C.C. Petty, J. Ferron, et al., Discharge improvement through control of neoclassical tearing modes by localized ECCD in DIII-D, Nucl. Fusion, 43, 2003, 1128.

[9] M. Reich, A. Bock, M. Maraschek and the ASDEX Upgrade Team, et al., NTM localization by correlation of Te and dB/dt, Fusion Sci. and Technol., 61, 2012, 309.

[10] M. Reich, L. Barrera-Orte, K. Behler, A. Bock, L. Giannone, M. Maraschek, et al., Europhysics Conference Abstracts (Proc. of the 39th EPS Conference on Plasma Physics, Stockholm, Sweden) 2012, http://ocs.ciemat.es/epsicpp2012pap/pdf/PD4.004.pdf.

[11] E. Poli, A.G. Peeters, and G.V. Pereverzev, TORBEAM, a beam tracing code for electron-cyclotron waves in tokamak plasmas, Comput. Phys. Commun., 136, 2001, 90.

[12] K. Nagasaki, A. Isayama, S. Ide, and JT-60 team, Stabilization effect of early ECCD on a neoclassical tearing mode in the JT-60U tokamak, Nucl. Fusion, 43, 2003, L7.

[13] R.J. La Haye, D.A. Humphreys, J.R. Ferron, T.C. Luce, W. Perkins, C.C. Petty, et al., Higher stable beta by use of pre-emptive electron cyclotron current drive on DIII-D, Nucl. Fusion, 45, 2005, L37.

[14] A. Mlynek, M. Reich, L. Giannone, W. Treuterrer, K. Behler, H. Blank, et al., Real-time feedback control of the plasma density profile on ASDEX Upgrade, Nucl. Fusion, 51, 2011, 043002.

[15] B.A. Hennen, E. Westerhof, P.W.J. M.Nuij, J.W. Oosterbeek, M.R. de Baar, W.A. Bongers, et al., Real-time control of tearing modes using a line-of-sight electron cyclotron emission diagnostic, Plasma Phys. Control. Fusion, 52, 2010, 104006. 
[16] W. A. Bongers, A. Goede, E. Westerhof, N.J. Doelman, F.C. Schüller, M.R. de Baar, et al., Magnetic island localization for NTM control by ECE viewed along the same optical path of the ECCD beam, Fusion. Sci. Technol., 55, 2009,188.

[17] K. Lackner, Computation of ideal MHD equilibria, Comput. Phys. Commun., 12, 1976, 33.

[18] J.D. Jackson, Classical Electrodynamics, J.Wiley and Sons, New York, 1999, 142.

[19] J. Simpson, J. Lane, C. Immer and R.Youngquist, Simple analytic expressions for the magnetic field of a circular current loop, 2001, http://ntrs.nasa.gov/archive/nasa/casi.ntrs.nasa.gov/20010038494_2001057024.pdf.

[20] M. Abramowitz and I.A. Stegun, Handbook of Mathematical Functions, Dover Publications, New York, 1972, 591.

[21] M. Abramowitz, and I.A. Stegun, Handbook of Mathematical Functions, Dover Publications, New York, 1972, 588.

[22] J.M. Moret, F. Buhlmann, D. Fasel, F. Hofmann and G.Tonetti, Magnetic measurements on the TCV tokamak, Rev. Sci. Instrum., 69,1998, 2333.

[23] L. Giannone, R. Fischer, J.C. Fuchs, O. Gruber, K. Lackner, P.J. McCarthy, et al., Europhysics Conference Abstracts (Proc. of the 37th EPS Conference on Plasma Physics, Dublin, Ireland), 2010, http://ocs.ciemat.es/EPS2010PAP/pdf/P4.122.pdf.

[24] STACC-HC Specifications, 2012, http://www.hitecups.com/import/assetmanager/0/350/STACC-HC-specs.pdf. 
[25] J.R. Ferron, M.L. Walker, L.L. Lao, H.E. St. John, D.A. Humphreys, J.A. Leuer, et al., Real time equilibrium construction for tokamak discharge control, Nucl. Fusion, 36, 1998, 1055.

[26] P.J. McCarthy, Identification of edge-localized moments of the current density profile in a tokamak equilibrium from external magnetic measurements, Plasma Phys. Control. Fusion, 54, 2012, 015100.

[27] T. Takeda and S.Tokuda Computation of MHD Equilibrium of Tokamak Plasma, J. Comput. Phys. 93, 1991, 1.

[28] W. Zwingmann, Equilibrium analysis of steady state tokamak discharges, Nucl. Fusion, 43, 2003, 842.

[29] G. Hommen, M. de Baar, J. Citrin, H.J. de Blank, R.J. Voorhoeve, M.F.M. de Bock, et al., A fast, magnetics-free flux surface estimation and q-profile reconstruction algorithm for feedback control of plasma profiles, Plasma Phys. Control. Fusion, 55, 2013, 025007

[30] R.W. Hockney and J.W. Eastwood, Computer simulation using particles, Taylor and Francis Group, New York, 1988.

[31] R. Preuss, R. Fischer, M. Rampp, K. Hallatschek, U. von Toissant, L.Giannone, et al., Parallel equilibrium algorithm for real time control of tokamak plasmas, Tech. rep., Max Planck Institute for Plasma Physics, Report IPP R/47, 2012.

[32] M.H. Hughes, Solution of Poisson's equation in cylindrical coordinates, Comput. Phys. Commun., 2, 1971, 157.

[33] W.H. Press, S.A. Teukolsky, W.T. Vetterling and B.P. Flannery, Numerical Recipes in C, 1999, Cambridge University Press, 50. 
[34] L. Giannone, R. Fischer, K. Lackner, Q. Ruan, A. Veermani, M. Cerna, et al., Proceedings of PCaPAC, Saskatoon, Saskatchewan, 2010 http://accelconf.web.cern.ch/accelconf/pcapac2010/papers/thpl024.pdf.

[35] W. Suttrop, J.C. Fuchs, R. Fischer, L. Giannone, A. Herrmann, R.M. McDermott, et al., Mitigation of edge localised modes with magnetic perturbations in ASDEX Upgrade, Fusion Eng. Des., 88, 2013, 446.

[36] B.L. Buzbee, A fast Poisson solver amenable to parallel computation, IEEE Trans. Comput., C-22, 1973, 793.

[37] P.N. Swarztrauber, The methods of cyclic reduction, Fourier analysis and the FACR algorithm for the discrete solution of Poisson's equation on a rectangle, SIAM Rev., 19, 1977, 490.

[38] P.N.Swarztrauber and R.A. Sweet, Vector and parallel methods for the direct solution of Poisson's equation, J. Comput. Appl. Math., 27, 1989, 241.

[39] M. Rampp, R. Preuss, R. Fischer, K. Hallatschek and L. Giannone et al., A parallel Grad-Shafranov solver for real-time control of tokamak plasmas, Fusion Sci. Technol., 62, 2012, 409.

[40] D.W. Swain and G.H. Nielsen, An efficient technique for magnetic analysis of non-circular, high-beta tokamak equilibria, Nucl. Fusion, 22, 1985, 1015.

[41] O. Barana, A. Murari, E. Joffrin, F. Sartori and Contributors to the JET-EFDA work programme al., Real-time calculation of plasma parameters for feedback control in JET, Nucl. Fusion, 44, 2004, 335.

[42] L.E. Zakharov and V.D. Shafranov, Equilibrium of a toroidal plasma with noncircular cross section, Sov. Phys. Tech. Phys., 18, 1973, 151. 
[43] V.D. Shafranov, Determination of the parameters betap and li in a tokamak for arbitrary shape of plasma pinch cross-section, Plasma Phys., 13, 1971, 757 .

[44] L.L. Lao, H.E. St. John, R.D. Stambaugh and W. Pfeiffer, Separation of betap and li in tokamaks of non-circular cross-section, Nucl. Fusion, 25, 1985, 1421.

[45] L.L. Lao, H.E. St. John, Q. Peng, J.R. Ferron, E.J. Strait, T.S. Taylor, et al., MHD equilibrium reconstruction in the DIII-D tokamak, Fusion Sci. Technol., 48, 2005, 968.

[46] B.W. Rice, K.H. Burrell, L.L. Lao and Y.R. Lin-Liu, Direct measurement of the radial electric field in tokamak plasmas using the Stark Effect, Phys. Rev. Lett., 79, 1997, 2694.

[47] D. Wróblewski and L.L. Lao, Polarimetry of motional Stark effect and determination of current profiles in DIII-D, Rev. Sci. Instrum., 63, 1992, 5140 .

[48] B.W. Rice, D.G. Nilson, K.H. Burrell and L.L. Lao, Simultaneous measurement of q and Er profiles using the motional Stark effect in highperformance DIII-D plasmas, Rev. Sci. Instrum., 70, 1999, 815.

[49] K. Ida, Experimental studies of the physical mechanism determining the radial electric field and its radial structure in a toroidal plasma, Plasma Phys. Control. Fusion, 40, 1998, 1429.

[50] J. Schirmer, G.D. Conway, H. Zohm, W. Suttrop, and the ASDEX Upgrade Team, et al., The radial electric field and its associated shear in the ASDEX Upgrade tokamak, Nucl. Fusion, 46, 2006, S780.

[51] W. Schneider, P.J. McCarthy, K. Lackner, O. Gruber, K. Behler, P. Martin, et al., ASDEX Upgrade MHD equilibria reconstruction on distributed workstations, Fusion Eng. Des., 48, 2000, 127. 
[52] W. Treutterer, L. Giannone, K.L. Lüddecke, G. Neu, G. Raupp, D. Zasche, et al., Real-time diagnostic integration with the ASDEX upgrade control system, Fusion Eng. Des., 84, 2009, 1871.

[53] J. Berrino, S. Cirant, F. Gandini, G. Granucci, E. Lazzaro, F. Jannone, et al., Automatic real-time tracking and stabilization of magnetic islands in a tokamak using temperature fluctuations and ECW power, IEEE Trans. Nucl. Sci., 53, 2006, 1009.

[54] C. Rapson, F. Monaco, M. Reich, J. Stober, W. Treutterer and the ASDEX Upgrade Team, et al., Simulation of feedback control system for NTM stabilisation in ASDEX Upgrade, Fusion Eng. Des., 88, 2013, 1137.

[55] L.L. Lao, J.R. Ferron, R.J. Groebner, W. Howl, H.St. John, E.J. Strait, et al., Equilibrium analysis of current profiles in tokamaks, Nucl. Fusion, 30, 1990, 1035.

[56] J.P. Meskat, H. Zohm, G. Gantenbein, S. Günter, M. Maraschek, W. Suttrop, et al., Analysis of the structure of neoclassical tearing modes in ASDEX Upgrade, Plasma Phys. Control. Fusion, 43, 2001, 1325.

[57] C. Ren, M. Chu, and J. Callen, Magnetic island deformation due to sheared flow and viscosity, Phys. Plasmas, 6, 1999, 1203. 


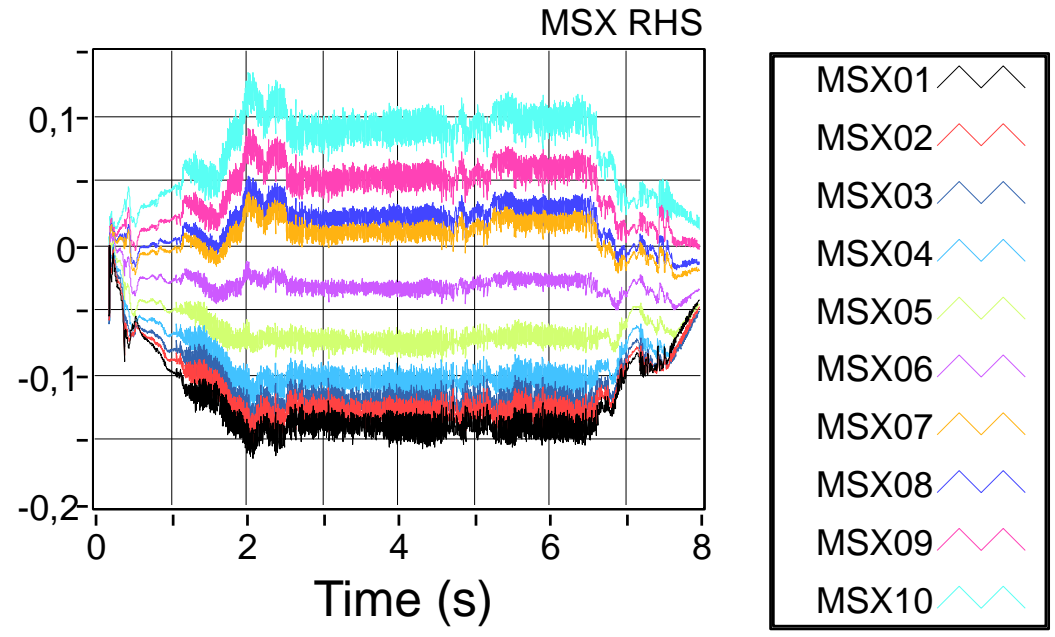

Figure 8: Time traces of the simulated right hand side of the response matrix for each MSE measurement volume.

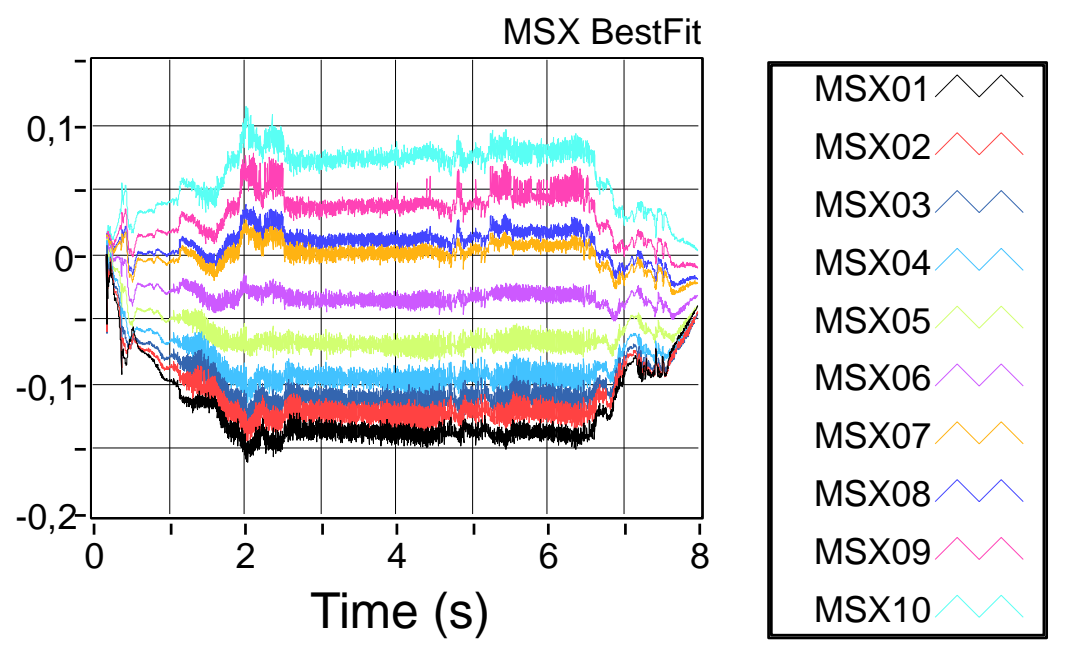

Figure 9: Time traces of the fitted right hand side of the response matrix for each MSE measurement volume. 


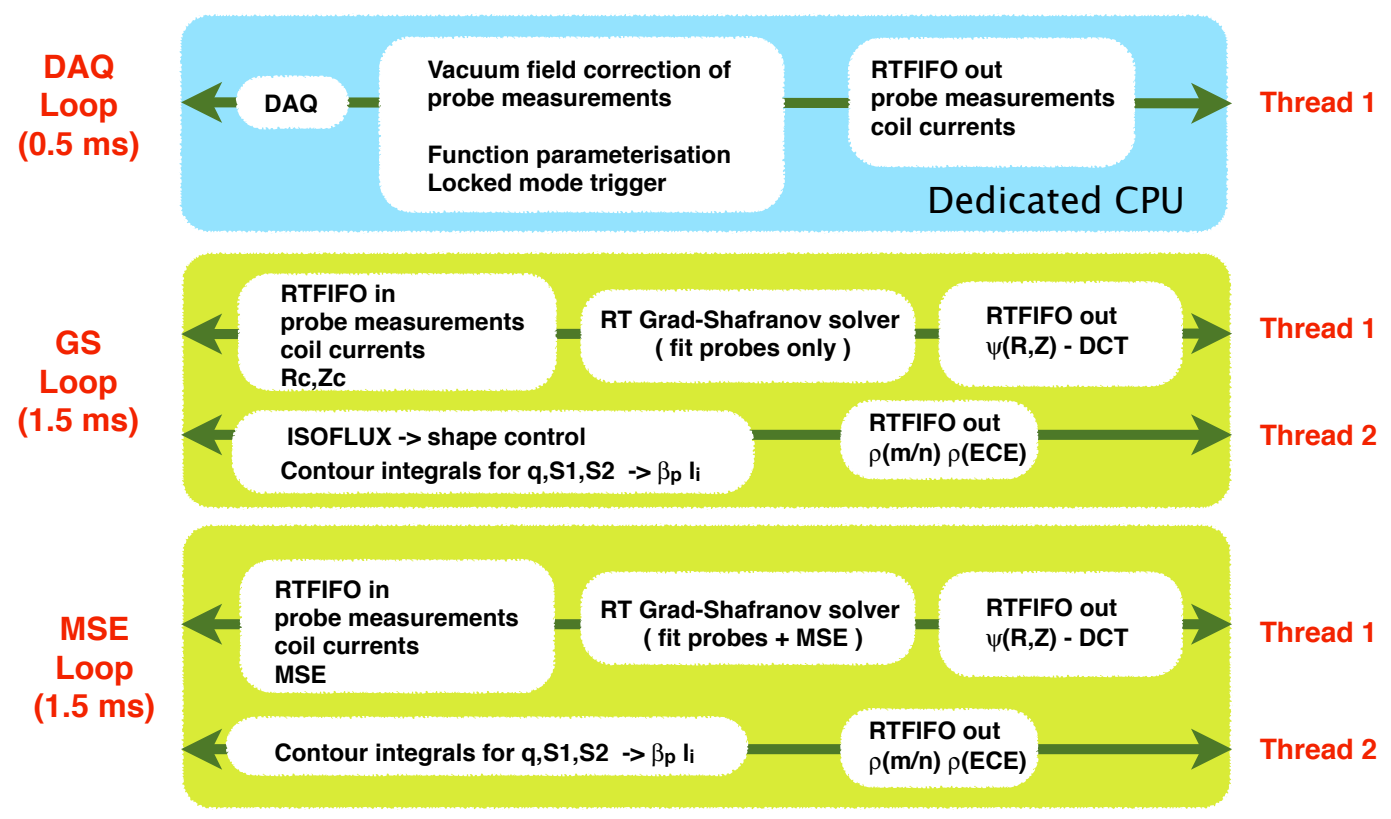

Figure 10: A diagram of the data acquisition application for calculating real-time magnetic equilibria on ASDEX Upgrade. The vacuum field correction of the probe measurements includes the contributions from the poloidal field coils and the magnetic perturbation coils. Minimum jitter of the cycle times was achieved by assigning a dedicated CPU to the data acquisition loop. 


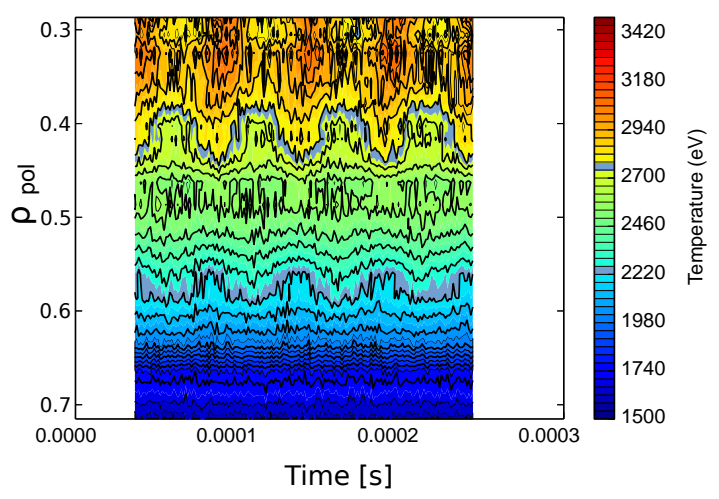

Figure 11: Contour plot of ECE temperature as a function of normalized radius in the presence of a NTM. In this discharge, the phase inversion of the temperature fluctuations can be distinctly observed at a normalized radius of 0.5 .

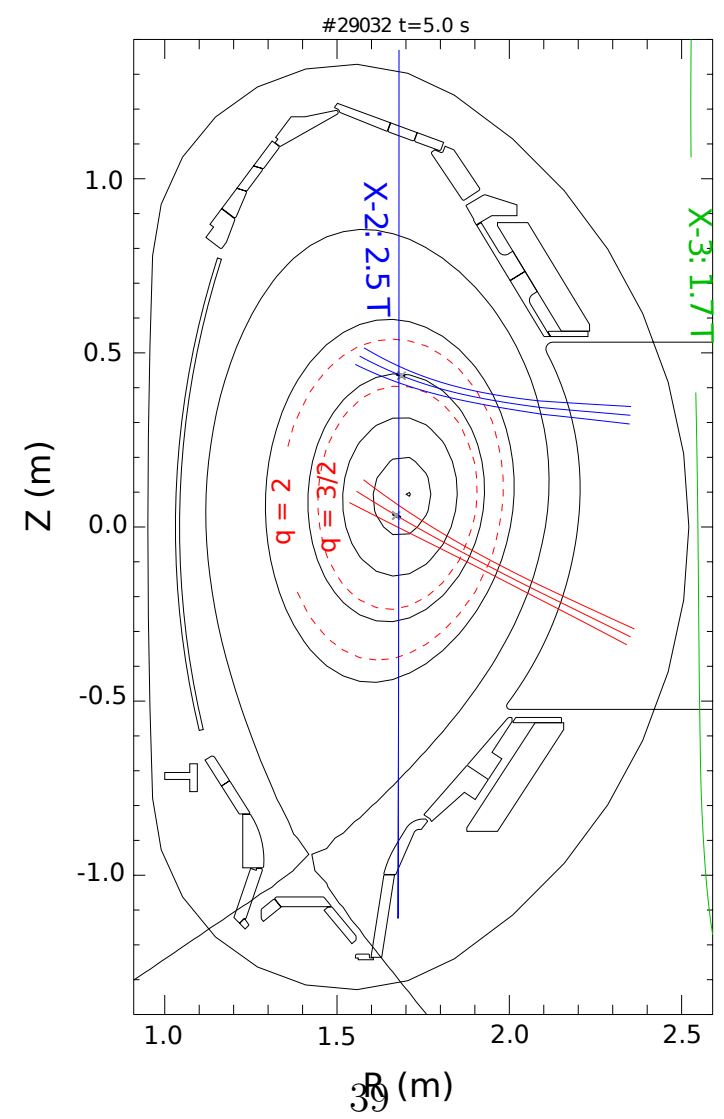

Figure 12: Microwave beam tracing for the pre-emptive NTM stabilization experiment with off-axis ECCD deposition ( blue) using the offline magnetic equilibrium. The $20 \mathrm{~ms}$ cycle time for the real-time version of TORBEAM is achieved by calculating the central ray only and having three parallel processes to know the change in ECCD deposition as a function of mirror launcher angle. 


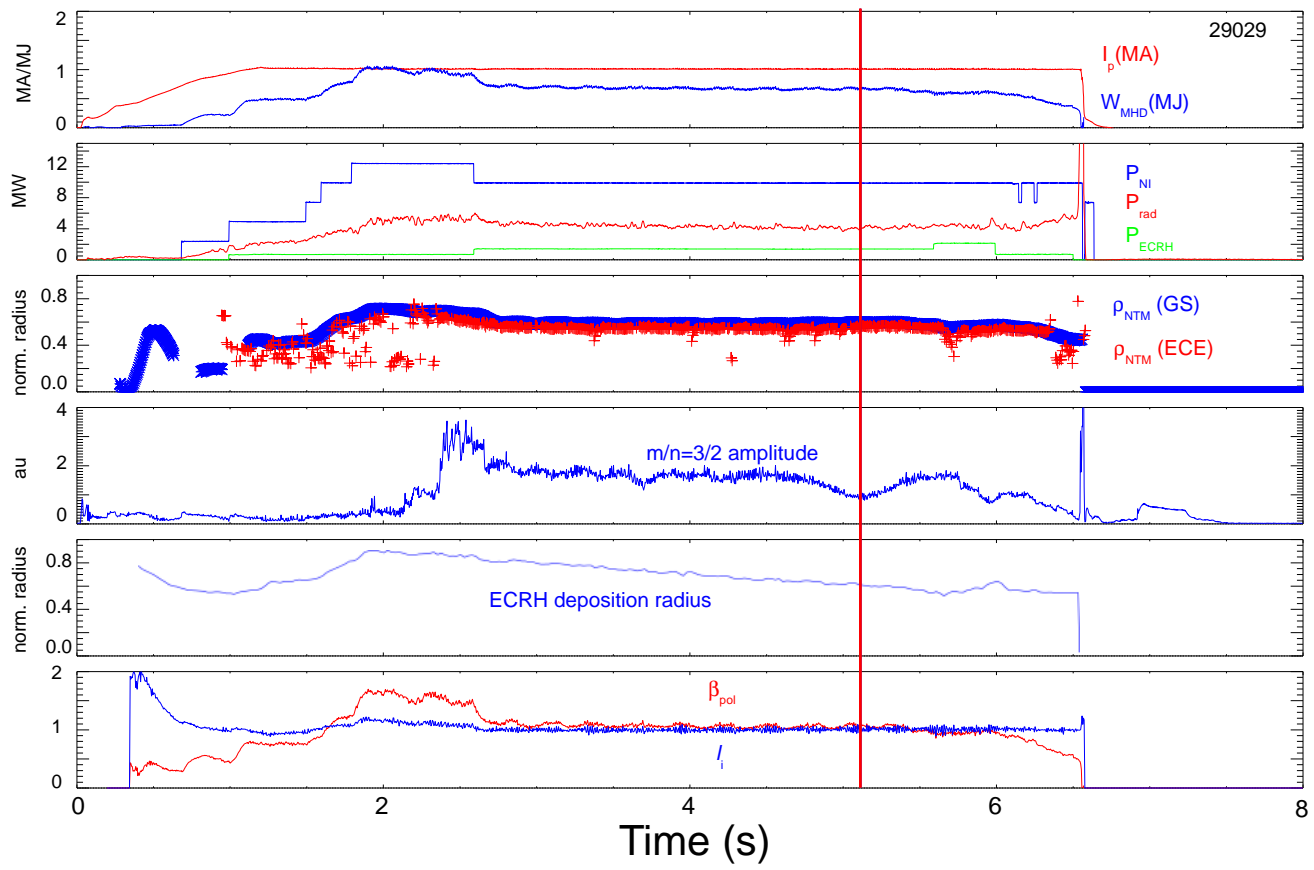

Figure 13: Time evolution of normalized radius of the 3/2 mode ( rhoNTM(GS)) predicted from the real-time magnetic equilibrium compared to that measured by ECE temperature fluctuations ( rhoNTM(ECE) ). From the magnetic fluctuation amplitude of the $\mathrm{m} / \mathrm{n}=$ $3 / 2$ mode, the NTM is present after $2.4 \mathrm{~s}$ while at $5.1 \mathrm{~s}$ a small dip corresponding to partial ECCD stabilization is indicated. 

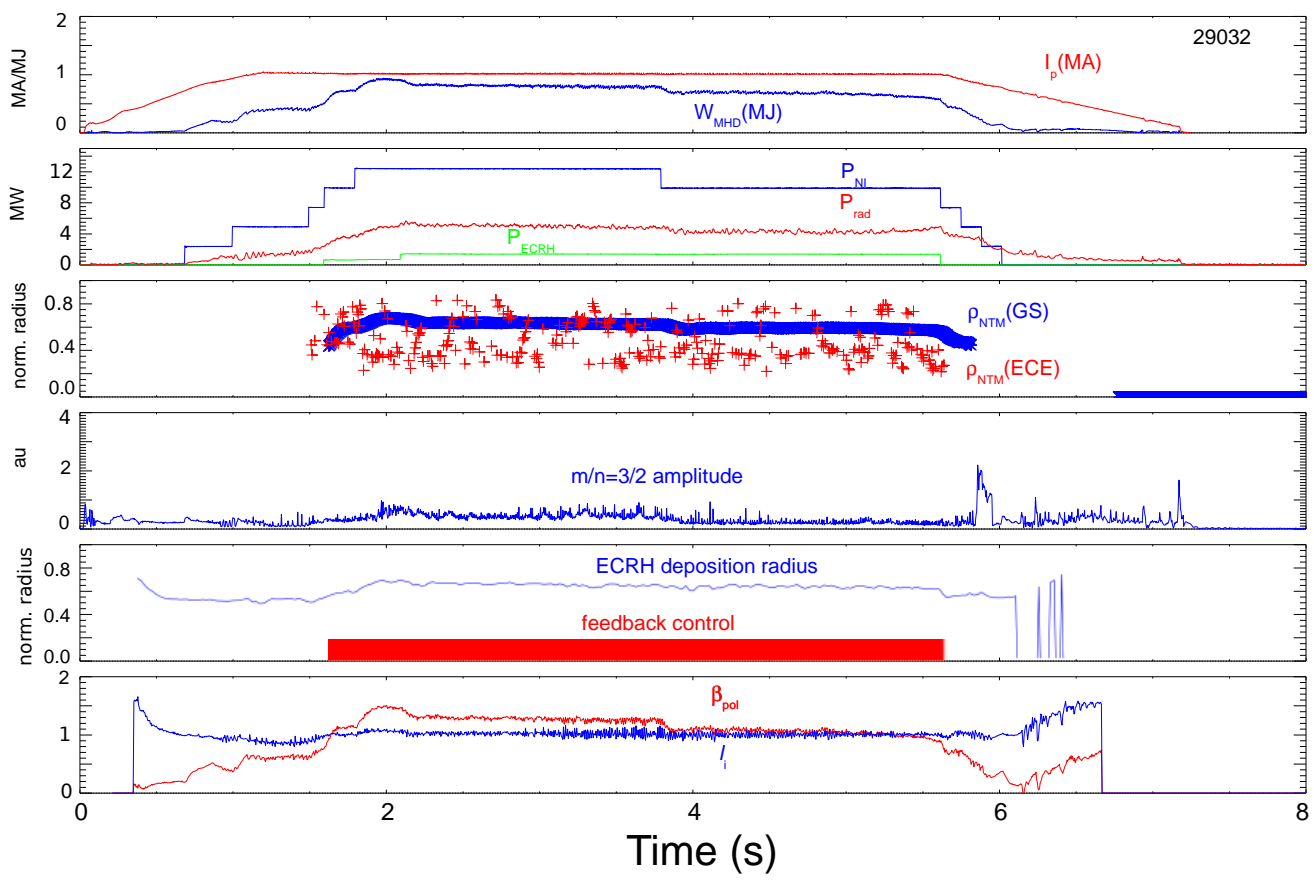

Figure 14: Pre-emptive stabilization of the 3/2 NTM mode. The mirror position is feedback controlled from the location of the normalized radius of the NTM calculated from the real-time magnetic equilibrium. Real-time calculations of microwave beam refraction are performed by the microwave beam tracing code, TORBEAM. The NTM cannot be localized as the temperature fluctuation amplitude of the NTM has been greatly reduced. Consequently, the normalized radius of the NTM measured by temperature fluctuations displays a large scatter. 

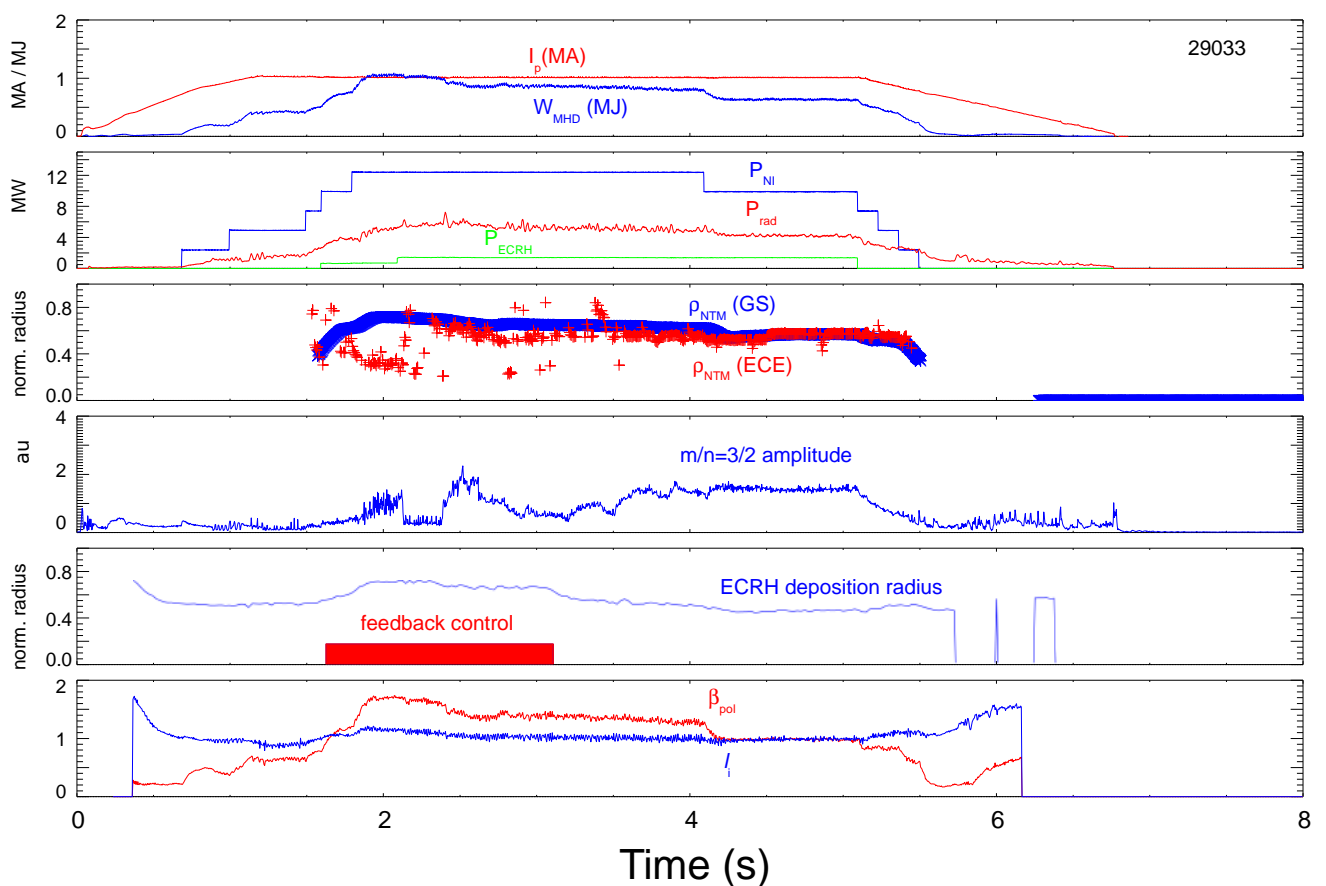

Figure 15: At $3.1 \mathrm{~s}$ the mirror feedback control is ended. After the mirror is moved away from the stabilizing position the NTM mode increases in amplitude. There is growth of the NTM mode after $2.4 \mathrm{~s}$ and a subsequent reduction in mode amplitude during feedback control. This indicates that pre-emptive stabilization from magnetic equilibria constrained only by probe measurements is not yet robust enough for routine operation. 


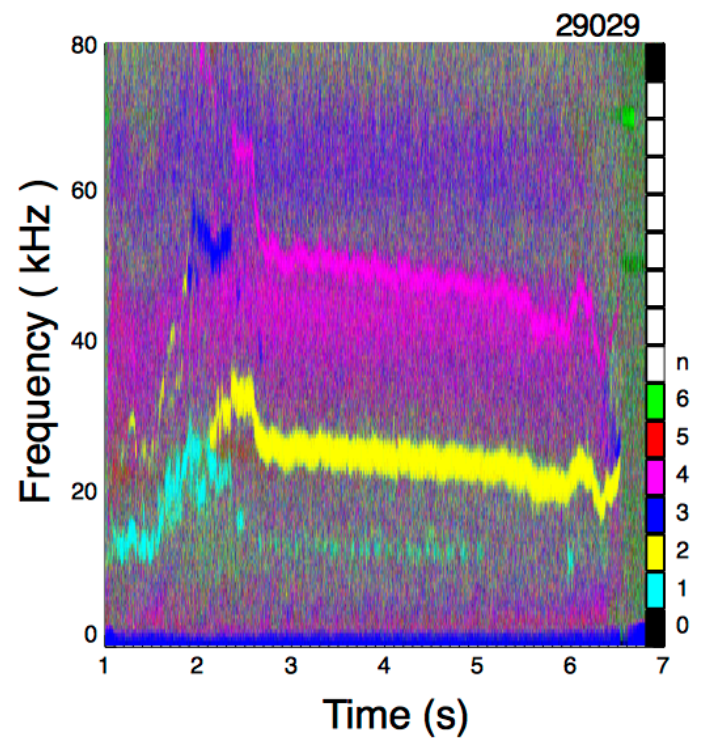

Figure 16: The legend on the right hand side of the plot is the colour code of the n number of the NTM. The $m / n=3 / 2$ mode dominates this discharge with $10 \mathrm{MW} N B I$ heating from $2.6 s$ without pre-emptive NTM stabilization.

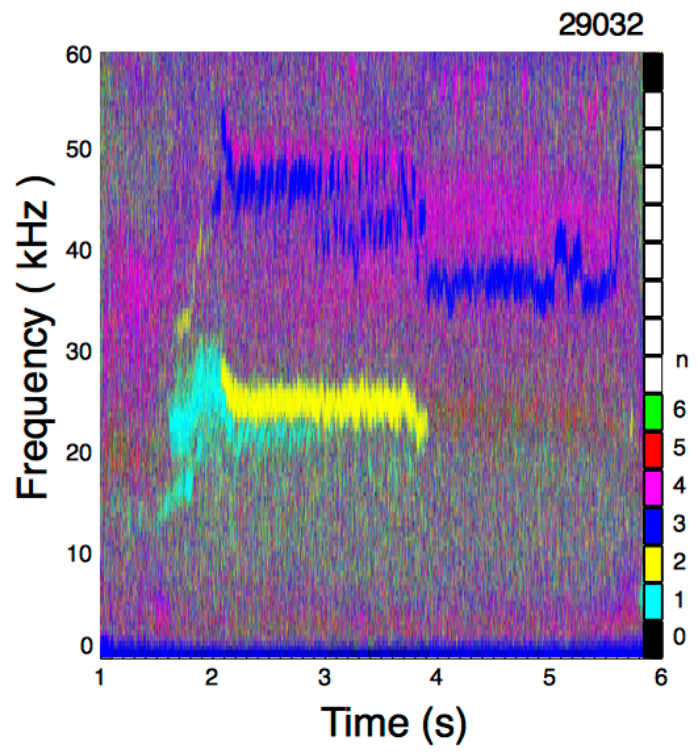

Figure 17: In this discharge with pre-emptive stabilization, a $m / n=3 / 2$ and $m / n=4 / 3$ mode are present in the phase up to $4 \mathrm{~s}$ with $12.5 \mathrm{MW}$ NBI heating. The NTM is only partially stabilized with ECCD power of $700 \mathrm{~kW}$. In the remainder of the discharge with $10 \mathrm{MW}$ NBI heating only a weak $\mathrm{m} / \mathrm{n}=4 / 3 \mathrm{NTM}$ is present and the $\mathrm{m} / \mathrm{n}=3 / 2 \mathrm{NTM}$ was fully suppressed. 


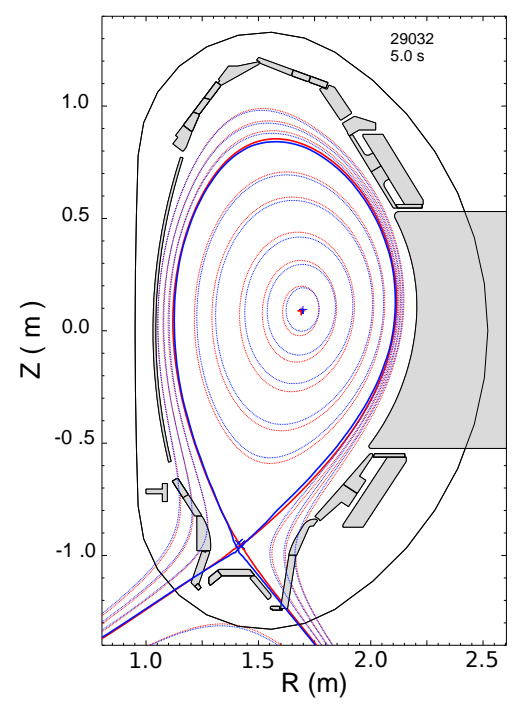

Figure 18: A comparison of the offline (red) and real-time magnetic equilibria (blue) for the discharge with pre-emptive stabilization. The number and form of the current basis functions are not the same, so that perfect agreement cannot be expected.

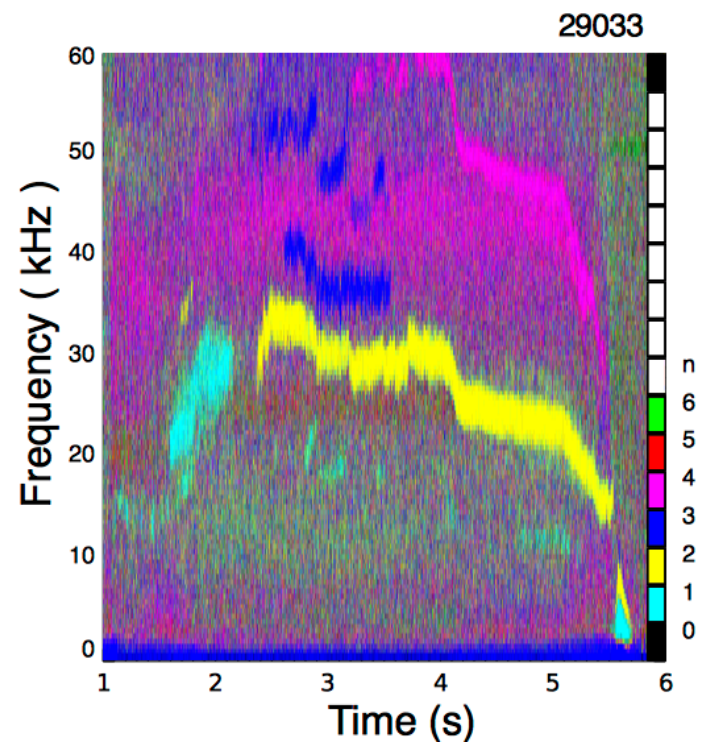

Figure 19: At $3.1 \mathrm{~s}$ the mirror feedback control is ended. After the mirror is moved away from the stabilizing position the NTM mode increases in amplitude. There is growth of the NTM mode after $2.4 \mathrm{~s}$ and a subsequent reduction in mode amplitude during feedback control. This indicates that pre-emptive stabilization from magnetic equilibria constrained only by probe measurements is not yet robust enough for routine operation. 